\title{
GAMMA-RAY LINES FROM SOLAR FLARES
}

\author{
R. R A M A TY \\ Laboratory for High Energy Astrophysics, NASA-Goddard Space Flight Center, Greenbelt, Md., U.S.A. \\ and \\ R. E. LINGENFELTER \\ Dept. of Planetary and Space Science, University of California, Los Angeles, Calif., U.S.A.
}

\begin{abstract}
We have treated in detail the theory of gamma-ray line production in solar flares. The strongest line, both predicted theoretically and detected observationally at $2.2 \mathrm{MeV}$, is due to neutron capture by protons in the photosphere. The neutrons are produced in nuclear reactions of flare accelerated particles which also produce positrons and prompt nuclear gamma rays. From the comparison of the observed and calculated intensities of the lines at 4.4 or $6.1 \mathrm{MeV}$ to that of the $2.2 \mathrm{MeV}$ line it is possible to deduce the spectrum of accelerated nuclei in the flare region; and from the absolute intensities of these lines it is possible to obtain the total number of accelerated nuclei at the Sun. The study of the $2.2 \mathrm{MeV}$ line also gives information on the amount of $\mathrm{He}^{3}$ in the photosphere. The study of the line at $0.51 \mathrm{MeV}$ resulting from positron annihilation complements the data obtained from the other lines; in addition it gives information on the temperature and density in the annihilation region and on the anisotropy of the accelerated electron beam which produces continuum gamma rays at energies greater than about $1 \mathrm{MeV}$.
\end{abstract}

\section{Introduction}

Measurements of accelerated charged particles near the Earth clearly indicate that such particles are produced in great profusion in solar flares. These particles consist of both electrons and nuclei; but until the advent of solar gamma-ray astronomy, observations in the radio and X-ray bands had revealed only the existence of the electronic component in the flare region itself.

In a previous paper (Lingenfelter and Ramaty, 1967) we treated in considerable detail the nuclear reactions produced by accelerated charged particles in solar flares and we showed that in large flares these reactions produce detectable lines in the gamma-ray region. We found that the strongest lines should be at $0.5,2.2,4.4$, and 6.1 MeV resulting from positron annihilation, neutron capture on hydrogen, and de-excitation of excited states in $\mathrm{C}^{12}$ and $\mathrm{O}^{16}$, respectively.

The recent observations by Chupp et al. (1973) of the first gamma-ray lines from solar flares confirm these predictions. During the flash phase of the 1972, August 4 flare all of these lines were observed with relative intensities essentially consistent with our calculations.

Since these observations became available, several additional studies on gamma-ray line production in solar flares have been undertaken. Ramaty and Lingenfelter (1973a) have investigated the consistency of the observations with the theory of nuclear reactions in flares; these authors also considered the effects of positronium formation and neutron propagation in the solar atmosphere (Ramaty and Lingenfelter, 1973b); Reppin et al. (1973) treated the time dependence of the $2.2 \mathrm{MeV}$ line; Wang and Ramaty (1974) have done a detailed calculation on neutron propagation and $2.2 \mathrm{MeV}$ 
line formation and they pointed out the importance of photospheric $\mathrm{He}^{3}$ as a nonradiative sink for the neutrons; Kozlovsky and Ramaty (1974a) have pointed out that $\alpha \alpha$ reactions produce the $\mathrm{Li}^{7}$ and $\mathrm{Be}^{7}$ lines at $478 \mathrm{keV}$ and $431 \mathrm{keV}$ which could be observable from flares; and Ramaty and Kozlovsky (1974) evaluated in detail the production of $\mathrm{H}^{2}, \mathrm{H}^{3}$ and $\mathrm{He}^{3}$ in flares and they attempted to deduce the number of protons released from the flare of 1972 , August 4 by combining the $\mathrm{He}^{3}$ and gammaray observations.

In the present paper we wish to summarize the above material and to present updated calculations on the production of gamma-ray lines in solar flares. In Section II we define the interaction models that we use in our calculations; in Section III we consider neutron production and $2.2 \mathrm{MeV}$ line formation; in Section IV we consider the production of prompt gamma-ray lines with special emphasis on the $4.4 \mathrm{MeV}$ and 6.1 MeV lines for which observational data exists; in Section V we compare the results of Sections III and IV with data for the 1972, August 4 flare and we deduce the number and spectrum of accelerated particles at the Sun; in Section VI we treat problems concerning the formation of the $0.51 \mathrm{MeV}$ line, and we summarize our results in Section VII.

\section{Interaction Models}

We consider two limiting interaction models (e.g. Ramaty and Lingenfelter, 1973a): A thin-target model in which the spectrum of accelerated particles is not modified during the time in which the nuclear interactions take place, and a thick-target model in which the accelerated particles move from the flare region downward into the Sun, undergoing nuclear interactions as they slow down in the solar atmosphere. In the thin-target model it is assumed that either the total path length traversed by the particles at the Sun is small in comparison with their interaction length, or that the particle energy loss from ionization and nuclear interactions is just balanced by energy gains from acceleration.

For the composition of the ambient solar atmosphere we use the abundances given by Cameron (1973). For the accelerated particle populations in both the thin- and thick-target models we consider power-law and exponential spectra. In the thin-target model these are

and

$$
N_{i}(E)=k_{i} E^{-s},
$$

$$
N_{i}(P)=k_{i}^{\prime} \exp \left(-P / P_{0}\right),
$$

respectively. Here $N_{i}(E)$ and $N_{\mathrm{i}}(P)$ are the instantaneous numbers of accelerated particles of kind $i$ in the interaction region per unit energy per nucleon, $E$, or unit rigidity, $P ; k_{i}$ and $k_{i}^{\prime}$ are constants determined by normalizing the $N_{i}$ 's to 1 proton of energy greater than $30 \mathrm{MeV}$ and by using the composition of the ambient solar atmosphere; and $s$ and $P_{0}$ are, respectively, the spectral index and characteristic rigidity assumed to be the same for all accelerated particle components. In the thick-target model we use expression similar to Equations (1) and (2), but we replace the instanta- 
neous numbers $N_{i}$ by total numbers, $\bar{N}_{i}$, such that $\bar{N}_{i}(N)$ and $\bar{N}_{i}(P)$ are the total number of accelerated charged particles per unit energy per nucleon or unit rigidity that are released from the flare region downward into the Sun. As with the instantaneous fluxes in the thin-target model, the normalizations of the $\bar{N}_{i}$ 's are determined by using the composition of the ambient solar atmosphere and 1 proton of energy greater than $30 \mathrm{MeV}$.

In the thin-target model, the production rate of secondaries from a particular reaction is given by

$$
q_{i}=n_{i} \int_{0}^{\infty} \mathrm{d} E N_{i}(E) c \beta \sigma_{i}(E),
$$

where $n_{i}$ is the number density of target atoms in the solar atmosphere, $c \beta$ is particle velocity, and $\sigma_{i}(E)$ is the cross-section as a function of energy per nucleon. The units of $q_{i}$ are secondary particles per second. In the thick-target model, the total production of secondary particles for a given reaction is

$$
Q_{i}=\eta_{i} \int_{0}^{x} \mathrm{~d} E^{\prime} \bar{N}_{i}\left(E^{\prime}\right) \int_{0}^{E^{\prime}} \mathrm{d} E \frac{\mathrm{d} x}{\mathrm{~d} E} \sigma_{i}(E)
$$

where $\eta_{i}$ is the number of target nuclei per gram of solar material, and $\mathrm{d} E / \mathrm{d} x$ is the stopping power of the primary particles in solar material due to both Coulomb and nuclear collisions. The quantities $Q_{i}$ are the total number of secondaries. Expressions similar to Equations (3) and (4) can be written down for rigidity spectra.

By inverting the order of integration, Equation (4) can be written as

$$
Q_{i}=\eta_{i} \int_{0} \mathrm{~d} E \frac{\mathrm{d} x}{\mathrm{~d} E} \sigma_{i}(E) \int_{0}^{s} \mathrm{~d} E^{\prime} \bar{N}_{i}\left(E^{\prime}\right) .
$$

Because the inner integral is just the integral spectrum of $\bar{N}_{i}(E)$, Equation (5) can be further simplified,

$$
Q_{i}=\eta_{i} \int_{0}^{\infty} \mathrm{d} E \frac{\mathrm{d} x}{\mathrm{~d} E} \sigma_{i}(E) \bar{N}_{i}(>E) .
$$

In our subsequent treatment of secondary particle production, we shall use Equations (3) and (6) or their equivalents for rigidity spectra.

\section{Neutron and 2.23 MeV Gamma-Ray Production}

Neutron production by accelerated charged particles was treated by Lingenfelter et al. (1965) and Lingenfelter and Ramaty (1967). Recently, Kozlovsky (1974, private communication) has updated the cross sections used by these authors and has added 
additional data at low energies including data on $\alpha$-particle induced reactions. The neutron production cross-sections are shown in Figure 1. Here pp. p $\alpha, \alpha \alpha$, pCNO and $\alpha \mathrm{CNO}$ indicate neutron production in proton-hydrogen, proton-helium, $\alpha$-particlehelium, proton-heavy nuclei, and $\alpha$-particle-heavy nuclei reactions, respectively. The latter two cross-sections are the neutron production cross-sections for all nuclei with $A \geqslant 12$, normalized to one such nucleus by using the elemental and isotopic abundances of Cameron (1973).

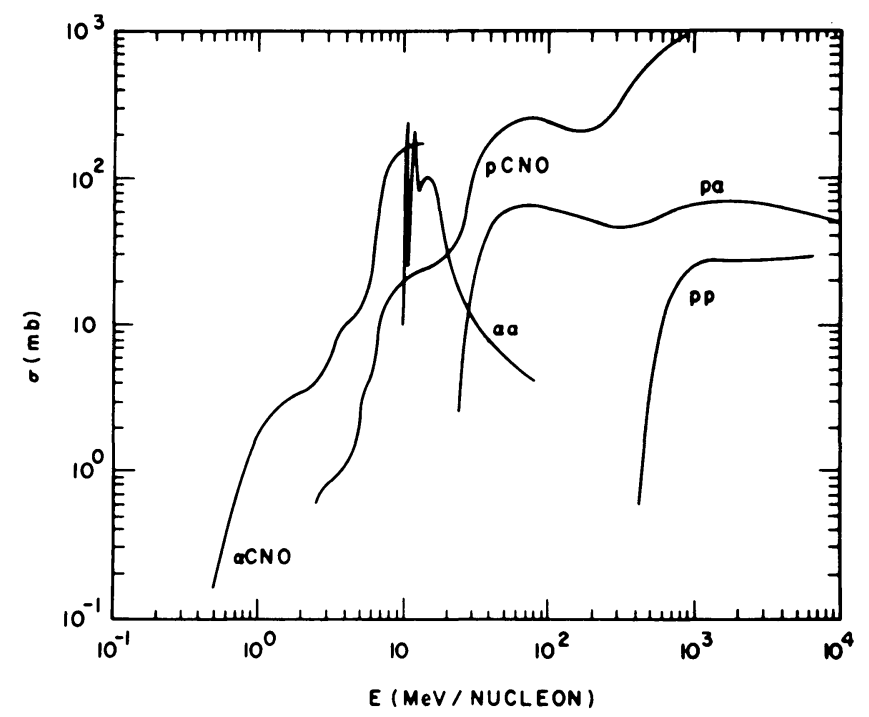

Fig. 1. Neutron production cross-sections.

The instantaneous neutron production rates in the thin-target model for power-law and exponential spectra are shown in Figures 2 and 3, respectively. The various production modes are: $p p$ (proton-hydrogen), $\mathrm{p} \alpha$ (proton-helium), $\alpha \mathrm{p}(\alpha$-particlehydrogen), $\alpha \alpha$ ( $\alpha$-particle-helium), pCNO (proton-heavy nuclei), CNOp (heavy nucleihydrogen), $\alpha \mathrm{CNO}$ ( $\alpha$-particle-heavy nuclei), and CNO $\alpha$ (heavy nuclei-helium). As can be seen for flatter spectra (smaller values of $s$ or larger values of $P_{0}$ ) the neutrons are produced mainly in $\mathrm{p} \alpha, \mathrm{pp}$ and $\alpha \mathrm{p}$ reactions. For power-law spectra (Figure 2 ) neutron production at large values of $s$ is mainly due to $\alpha \mathrm{CNO}$ and $\mathrm{CNO} \alpha$ reactions; the contribution of pCNO and CNOp reactions is small at all values of $s$; and $\alpha \alpha$ reactions make a major contribution around $s=4$. For exponential spectra, (Figure 3) almost all of the neutrons are produced in $\mathrm{p} \alpha$ reactions at most values of $P_{0}$. In this case the relative contributions of reactions induced by $\alpha$-particles and heavy nuclei are lower than for power-law spectra. Because particles with $Z \geqslant 2$ have larger rigidities than protons, it follows from Equation (2) that their fluxes relative to the proton flux at the same energy per nucleon is lower than in the power-law case given by Equation (1). Since the nuclear cross-sections are the same for the direct and inverse reactions 


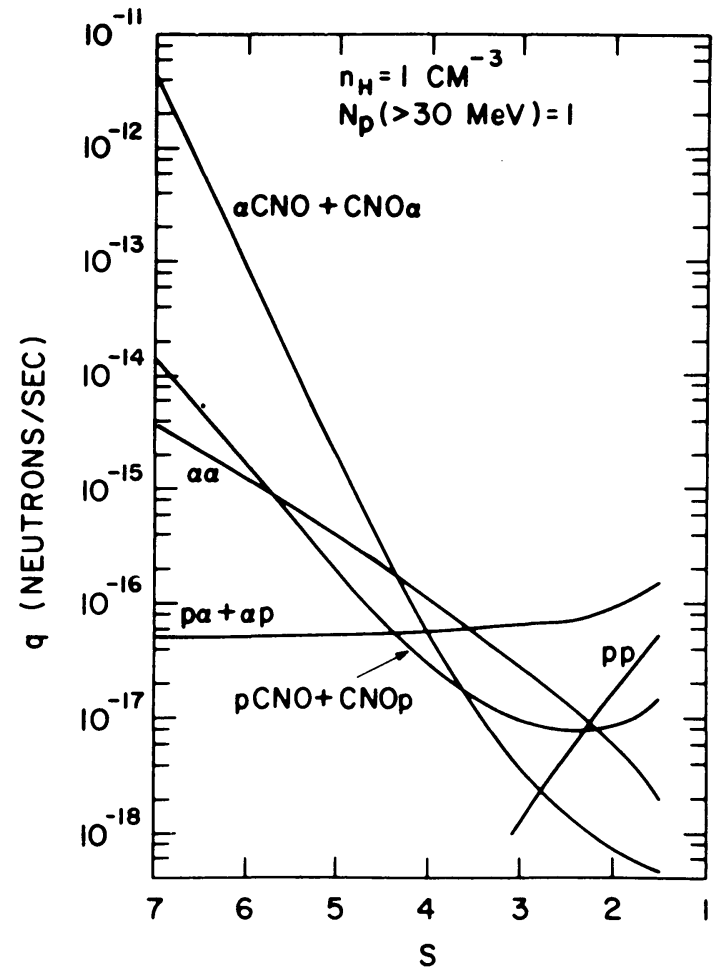

Fig. 2. Partial neutron production modes in the thin-target model with power-law spectra.

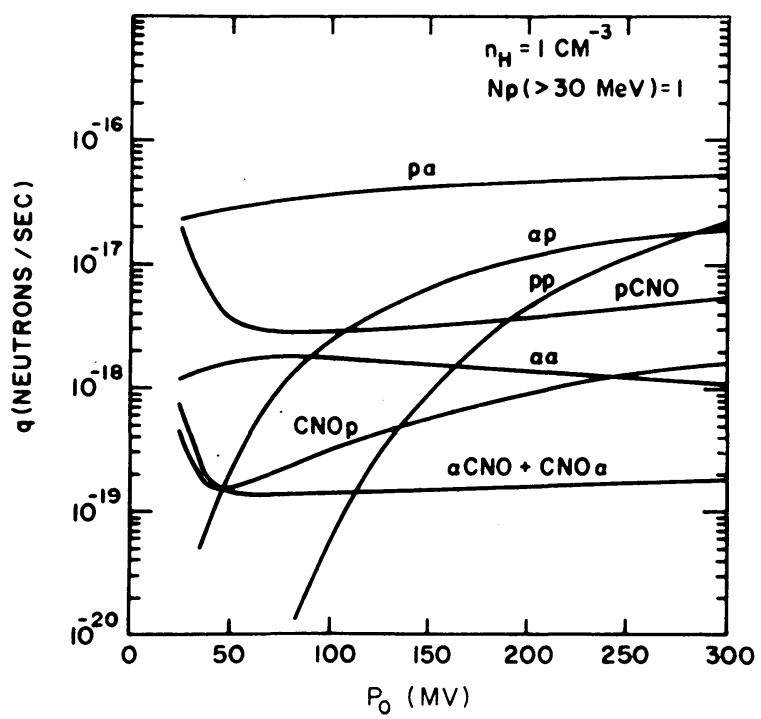

Fig. 3. Partial neutron production modes in the thin-target model with exponential spectra. 
at the same energy per nucleon, for particle spectra which are exponential in rigidity more neutrons are produced by a proton induced reaction than by the corresponding inverse process.

The total neutron production rates in the thin-target model, $q$, and the total neutron yields in the thick-target model, $Q$, are shown in Figure 4 for power-law and exponential spectra. In the thin-target model, for flat primary spectra the neutron production rates are about the same for the exponential and power-law cases. This result is due

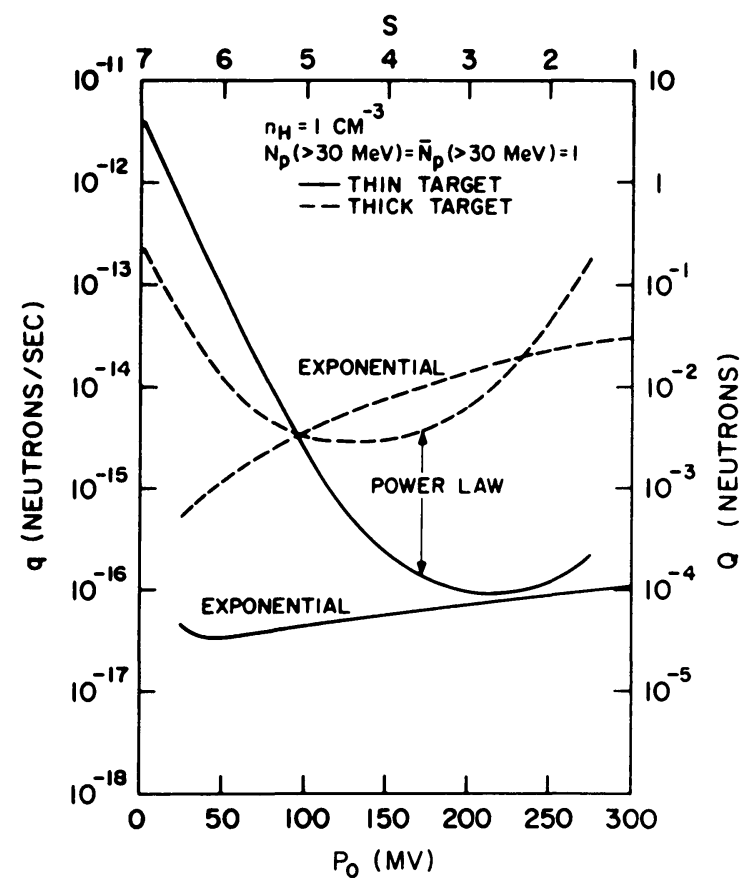

Fig. 4. Total neutron production in the thin- and thick-target models with power-law and exponential spectra.

simply to the fact that for such spectra most of the neutrons are produced in $\mathrm{p} \alpha$ reactions with effective threshold around $30 \mathrm{MeV}$ nucleon $^{-1}$ and all of the assumed spectra are normalized to 1 proton above this energy. But because a power law spectrum contains a much larger number of low-energy particles than an exponential spectrum with this same normalization, the steep power law spectrum can yield orders of magnitude more neutrons from $\alpha \mathrm{CNO}$ and $\mathrm{CNO} \alpha$ reactions which have thresholds more than an order of magnitude below $30 \mathrm{MeV}$ nucleon $^{-1}$.

Similar effects are evident also in the thick-target model except that here the relative contribution of the low energy particles in general is diminished because of their shorter range.

Having considered the production of neutrons, let us now discuss their propagation and the ensuing gamma-ray line production. Wang and Ramaty (1974) considered in 
detail the effects of neutron propagation in the solar atmosphere on the production of gamma rays by the reaction

$$
\mathrm{n}+\mathrm{p} \rightarrow \mathrm{d}+\gamma
$$

In their treatment a distribution of neutrons was released in the chromosphere or corona, and the path of each neutron after its release was followed by a computer Monte-Carlo simulation. If the neutrons are released above the photosphere, any initially upward moving neutron escapes from the Sun. Some of the downward moving neutrons can also escape after being backscattered elastically by ambient protons, but most of these neutrons either are captured or decay at the Sun. Because the probability for elastic scattering is much larger than the capture probability, the majority of the neutrons are thermalized before they get captured. Since the thermal speed in the photosphere (where most of the captures take place) is much smaller than the speed of light, the gamma-rays from reaction (7) are essentially all at $2.2 \mathrm{MeV}$ and the Doppler-broadened width of this line is negligible.

The bulk of neutrons at the Sun are captured either on $\mathrm{H}$ or on $\mathrm{He}^{3}$. Whereas capture on $\mathrm{H}$ yields a $2.2 \mathrm{MeV}$ photon, capture on $\mathrm{He}^{3}$ proceeds via the radiationless transition

$$
\mathrm{n}+\mathrm{He}^{3} \rightarrow \mathrm{H}^{3}+\mathrm{p}
$$

and hence produces no photons. The cross-sections for reactions (7) and (8) are $2.2 \times 10^{-30} \beta^{-1} \mathrm{~cm}^{2}$ and $3.7 \times 10^{-26} \beta^{-1} \mathrm{~cm}^{2}$, respectively, where $\beta$ is the velocity of the neutron (for details see Wang and Ramaty, 1974). Thus if the $\mathrm{He}^{3} / \mathrm{H}$ ratio in the photosphere is $\sim 5 \times 10^{-5}$ comparable to that observed in the solar wind, nearly equal numbers of neutrons are captured on $\mathrm{He}^{3}$ as on $\mathrm{H}$.

The results of the Monte-Carlo calculations of Wang and Ramaty (1974) are presented in Figures 5 and 6 for two assumptions on the photospheric $\mathrm{He}^{3}$ abundance: $\mathrm{He}^{3} / \mathrm{H}=0$ and $\mathrm{He}^{3} / \mathrm{H}=5 \times 10^{-5}$. In these calculations an isotropic distribution of monoenergetic neutrons of energy $E_{\mathrm{n}}$ is released above the photosphere. The solid lines are the probabilities for the various indicated processes. As can be seen, the capture and loss probabilities increase with increasing energy, because higher energy neutrons penetrate deeper into the photosphere. This reduces their escape probability and leads to a shorter capture time, thereby reducing the decay probability. When $\mathrm{He}^{3} / \mathrm{H}=$ $=5 \times 10^{-5}$, the probability for loss on $\mathrm{He}^{3}$ almost equals the capture probability on protons. The escape probability is greater than 0.5 , because all initially upward moving neutrons escape from the Sun. Note that the sum of all probabilities equals 1.

The dashed lines in Figures 5 and 6 are photon yields per neutron, $f\left(\theta, E_{\mathrm{n}}\right)$, for various neutron energies, $E_{\mathrm{n}}$, and angles, $\theta$, between the Earth-Sun line and the vertical to the solar surface. The function $f$ is defined such that for an average neutron production rate, $q$, the average $2.2 \mathrm{MeV}$ photon flux at Earth is

$$
\phi(2.2 \mathrm{MeV})=q f /\left(4 \pi R^{2}\right),
$$

where $R=1 \mathrm{AU}$. 


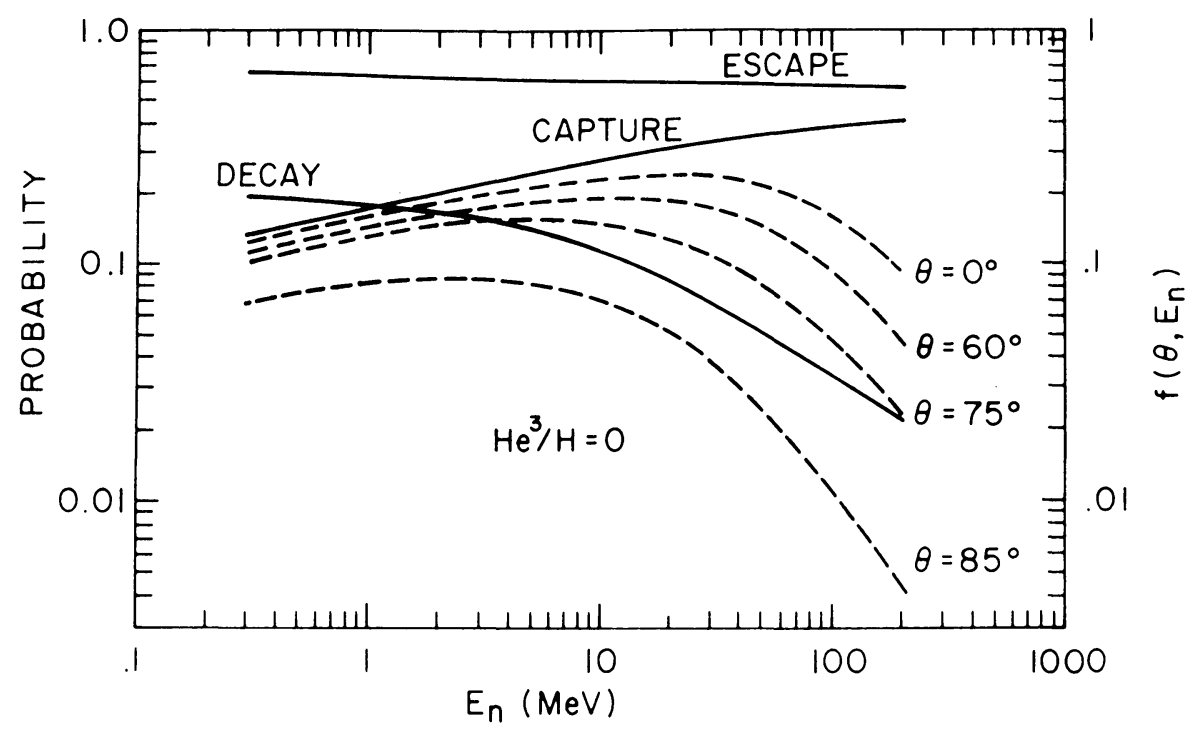

Fig. 5. Probabilities for neutron escape,decay, and capture in the solar atmosphere (solid lines), and photon yields per neutron (dashed lines) for no $\mathrm{He}^{3}$ in the photosphere.

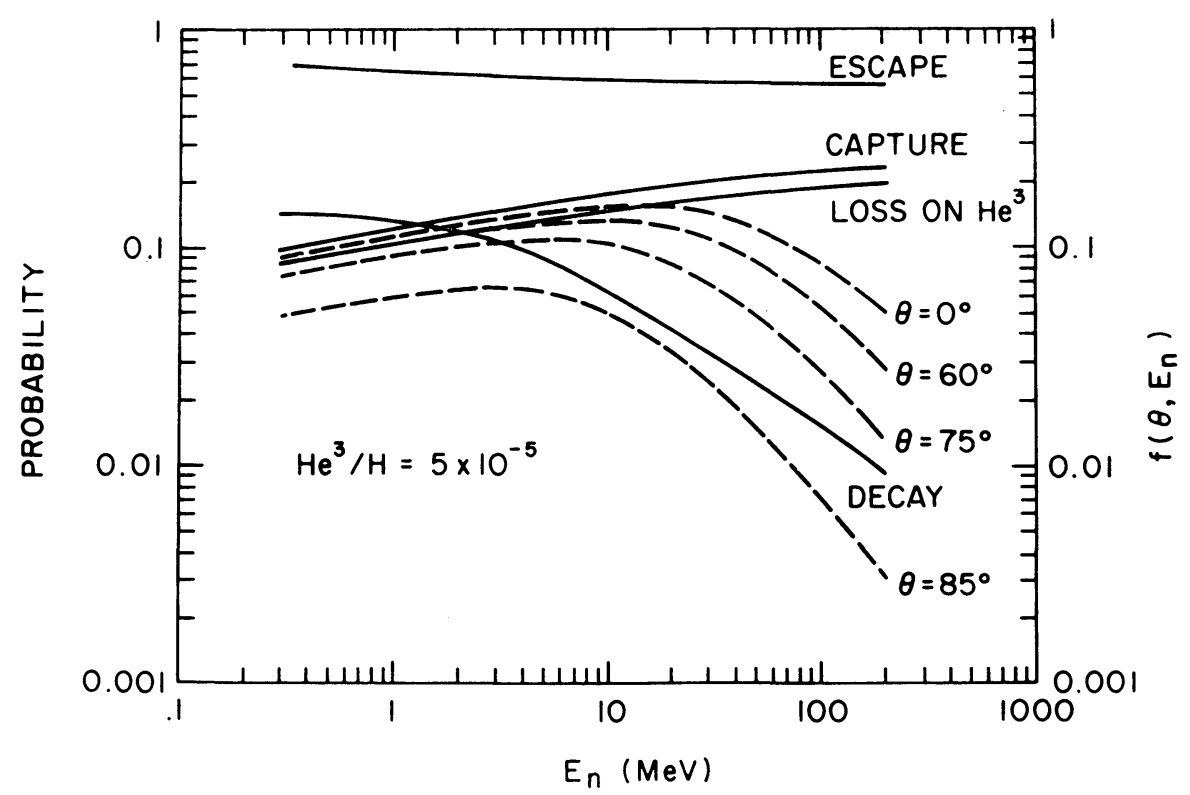

Fig. 6. Probabilities for neutron escape, decay, and capture in the solar atmosphere (solid line), and photon yields per neutron (dashed lines) for $\mathrm{He}^{3} / \mathrm{H} \quad 5 \cdots 10^{-5}$. 
At low neutron energies and $\theta$ near zero, $f$ is close to the capture probability on protons. This means that gamma rays from low-energy neutrons observed close to the vertical escape essentially unattenuated from the Sun. At higher energies and at larger angles, however, there is significant attenuation of the gamma rays due to Compton scattering in the photosphere. Even though $f$ does depend on $E_{\mathrm{n}}$, for flares sufficiently close to longitude and latitude zero on the Sun and neutron energies between about 1 and $100 \mathrm{MeV}$, we can approximate it by a constant. Most of the neutrons have energies in this range (Lingenfelter and Ramaty, 1967). Thus, for $\mathrm{He}^{3} / \mathrm{H} \simeq$ $\simeq 5 \times 10^{-5}$ we use $f \simeq 0.12$, and for $\mathrm{He}^{3} / \mathrm{H} \simeq 0$, we take $f \simeq 0.2$. Note that these approximations are quite valid for the flare of 1972, August 4, since its solar longitude and latitude where E08 and N14.

It should be noted that Equation (9) is valid for the average neutron flux only, because the instantaneous $2.2 \mathrm{MeV}$ flux lags behind the instantaneous neutron production rate. This lag is almost entirely due to the finite neutron capture time in the photosphere. Wang and Ramaty (1974) have investigated this effect, and some of their results are given in Table I. Here $\langle n\rangle$ is the most probable density in the photo-

\section{TABLE I}

Most probable neutron capture densities, capture times, $\tau_{c}$, and $\lambda=\tau_{\mathrm{c}}{ }^{-1}+\tau_{\mathrm{d}}{ }^{-1}$, where $\tau_{\mathrm{d}}$ is the neutron mean life.

\begin{tabular}{ccccccc} 
& & $\tau_{\mathrm{c}}(\mathrm{s})$ & & $\lambda^{-1}(\mathrm{~s})$ & \\
\cline { 3 - 5 }$E_{\mathrm{n}}(\mathrm{MeV})$ & $n>\left(\mathrm{cm}^{-3}\right)$ & $\mathrm{He}^{3} / \mathrm{H}=5 \times 10^{-5}$ & $\mathrm{He}^{3} / \mathrm{H}=0$ & $\mathrm{He}^{3} / \mathrm{H}=5 \times 10^{-5}$ & $\mathrm{He}^{3} / \mathrm{H}=0$ \\
1 & $7 \times 10^{16}$ & 119 & 214 & 105 & 173 \\
10 & $1.2 \times 10^{17}$ & 69 & 125 & 64 & 110 \\
100 & $3 \times 10^{17}$ & 28 & 50 & 27 & 47
\end{tabular}

sphere where the captures take place, $\tau_{\mathrm{c}}$ is the mean capture time, and $\tau_{\mathrm{d}}$ is the neutron decay mean life. In terms of the parameter $\lambda=\tau_{c}^{-1}+\tau_{d}^{-1}$, the time profile of the 2.2 $\mathrm{MeV}$ photon flux from a monoenergetic burst of neutrons released at $t_{0}$ can be approximated (Wang and Ramaty, 1974) by

$$
\phi(2.2 \mathrm{MeV}) \propto \exp \left[-\lambda\left(t-t_{0}\right)\right]
$$

\section{Prompt Gamma-Ray Lines Production}

The various prompt gamma-ray lines that can be produced in solar flares together with their production mechanisms are listed in Table II. These lines have already been discussed by Ramaty and Lingenfelter (1973a) except for the $\mathrm{Li}^{7}$ and $\mathrm{Be}^{7}$ lines at $478 \mathrm{keV}$ and $431 \mathrm{keV}$. The possibility of producing these lines in $\alpha \alpha$ reactions has been pointed out recently by Kozlovsky and Ramaty (1974a) 


\section{TABLE II}

Prompt gamma-ray lines

$\begin{array}{lll}\begin{array}{l}\text { Photon energy } \\ (\mathrm{MeV})\end{array} & \text { Origin } & \text { Production mode } \\ 0.431 & & \\ 0.478 & \mathrm{Be}^{7 *} \text { de-excitation } & \mathrm{He}^{4}(\mathrm{\gamma}, \mathrm{n}) \mathrm{Be}^{7 * 0.431} \\ 1.63 & \mathrm{Li}^{7 *} \text { de-excitation } & \mathrm{He}^{4}(\mathrm{p}, \mathrm{p}) \mathrm{Li}^{7 * 0.478} \\ & \mathrm{Ne}^{20 * 1.63} \text { de-excitation } & \mathrm{Ne}^{20}\left(\mathrm{p}, \mathrm{p}^{\prime}\right) \mathrm{Ne}^{20 * 1.63} \\ & \mathrm{~N}^{14 * 3.94} \rightarrow \mathrm{N}^{14 * 2.31} \text { de-excitation } & \mathrm{N}^{14}\left(\mathrm{p}, \mathrm{p}^{\prime}\right) \mathrm{N}^{14 * 3.94} \\ & \mathrm{~N}^{14 * 2.31} \text { de-excitation } & \mathrm{N}^{14}\left(\mathrm{p}, \mathrm{p}^{\prime}\right) \mathrm{N}^{14 * 2.31} \\ & & \mathrm{~N}^{14}\left(\mathrm{p}, \mathrm{p}^{\prime}\right) \mathrm{N}^{14 * 3.94} \rightarrow \mathrm{N}^{14 * 2.31} \\ & & \mathrm{~N}^{14}(\mathrm{p}, \mathrm{n}) \mathrm{O}^{14} \rightarrow \mathrm{N}^{14 * 2.31} \\ 4.43 & \mathrm{C}^{12 * 4.43} \text { de-excitation } & \mathrm{C}^{12}\left(\mathrm{p}, \mathrm{p}^{\prime}\right) \mathrm{C}^{12 * 4.43} \\ & & \mathrm{C}^{12}\left(\alpha, \mathrm{x}^{\prime}\right) \mathrm{C}^{12 * 4.43} \\ 5.2 & & \mathrm{O}^{16}(\mathrm{p},-) \mathrm{C}^{12 * 4.42} \\ & & \mathrm{O}^{16}(\mathrm{p},-) \mathrm{O}^{15 * 5.20} \\ 6.14 & \mathrm{O}^{15 * 5.26} \text { de-excitation } & \mathrm{O}^{16}(\mathrm{p},-) \mathrm{N}^{15 * 5.28} \\ 7.12 & \mathrm{~N}^{15 * 5.28} \text { de-excitation } & \mathrm{O}^{16}\left(\mathrm{p}, \mathrm{p}^{\prime}\right) \mathrm{O}^{16 * 6.14} \\ & \mathrm{O}^{16 * 6.14} \text { de-excitation } & \mathrm{O}^{16}\left(\alpha, \alpha^{\prime}\right) \mathrm{O}^{16 * 6.14} \\ & & \mathrm{O}^{16}\left(\mathrm{p}, \mathrm{p}^{\prime}\right) \mathrm{O}^{16 * 7.12}\end{array}$

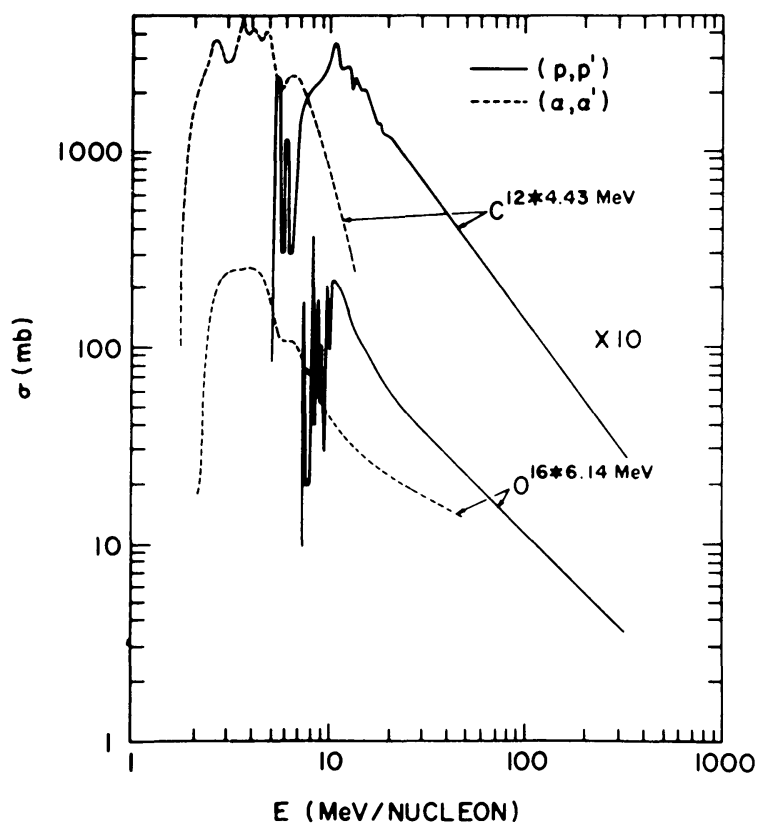

Fig. 7. $\mathrm{C}^{12 *}$ and $\mathrm{O}^{16 *}$ production cross-sections.

The cross-sections for the reactions $C^{12}\left(p, p^{\prime}\right) C^{12 * 4.43}, C^{12}\left(\alpha, \alpha^{\prime}\right) C^{12 * 4.43}$, $\mathrm{O}^{16}\left(\mathrm{p}, \mathrm{p}^{\prime}\right) \mathrm{O}^{16 * * 6.14}$, and $\mathrm{O}^{16}\left(x, \alpha^{\prime}\right) \mathrm{O}^{16 * 6.14}$ are given in Figure 7 . The cross-section for the proton induced reactions were summarized by Lingenfelter and Ramaty (1967), and the cross-sections for the $x$-particle induced reactions are from Kozlovsky 
(private communication). In addition, we also consider the reaction $\mathrm{p}+\mathrm{O}^{16} \rightarrow \mathrm{C}^{12 * 4.43}$ $+\cdots$ which was discussed previously (Ramaty and Lingenfelter, 1973a). By using these cross-sections and the interaction models discussed above, we can calculate the production rates of $\mathrm{C}^{12 *}$ and $\mathrm{O}^{16 *}$ and the resultant prompt photons at $4.43 \mathrm{MeV}$ and $6.14 \mathrm{MeV}$. We must distinguish, however, between reactions induced by accelerated protons or $\alpha$-particles, and reactions induced by accelerated heavy nuclei. For the former, the Doppler widths of the lines are small in comparison with available instrumental resolutions (about $150 \mathrm{keV}$ ). But for the latter the lines are significantly broadened by the motion of the excited fast nucleus which has lost little kinetic energy in the interaction. Because these lines are so broad that they cannot be resolved from the background with presently available instrumentation, in our treatment we consider the intensities of the $4.43 \mathrm{MeV}$ and $6.14 \mathrm{MeV}$ lines from proton and $\alpha$ particle induced reactions only.

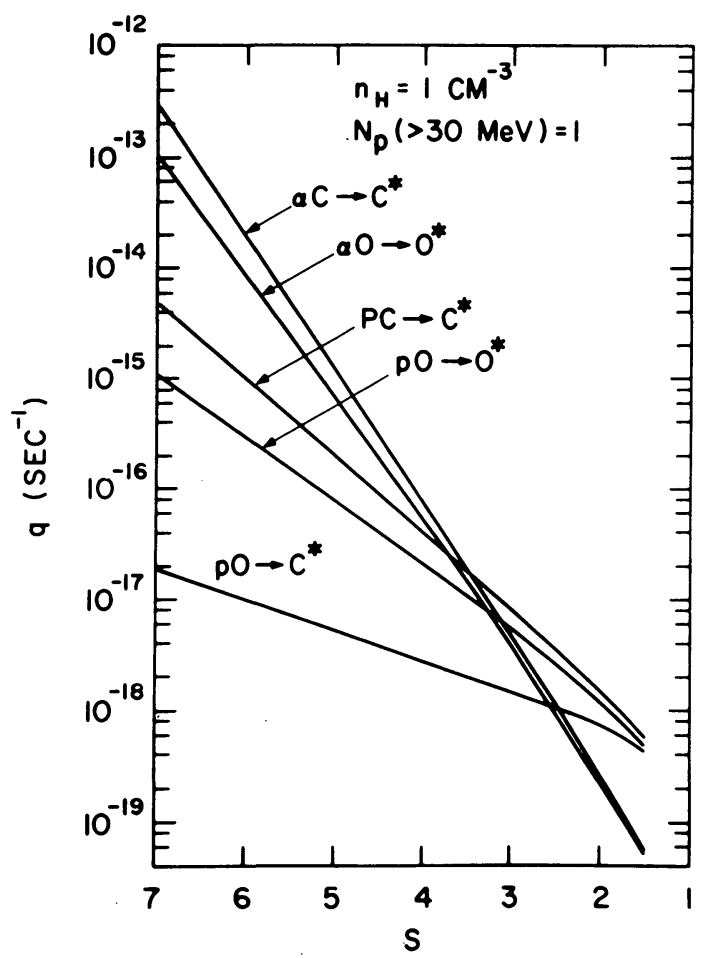

Fig. 8. $\mathrm{C}^{12 *}$ and $\mathrm{O}^{16 *}$ partial production modes in the thin-target model with power-law spectra.

The production rates of $\mathrm{C}^{12 *}$ and $\mathrm{O}^{16 *}$ in the thin-target model with power-law spectra are shown in Figure 8. We see that for flat spectra, the lines at 4.43 and $6.14 \mathrm{MeV}$ are produced mainly by proton-induced reactions, whereas for steep spectra (large values of $s$ ), the contributions of the $\alpha$-particles becomes important. For exponential spectra, proton-induced reactions are the principle source of the excited states at all values of $P_{0}$. 
The cross-section for $\mathrm{Li}^{7}$ production in $\alpha \alpha$ reactions is shown in Figure 9 (Kozlovsky and Ramaty, 1974b). The cross section for $\mathrm{Li}^{7 *}$ production is about half of the total $\mathrm{Li}^{7}$ production independent of energy. Similarly, the cross-section for $\mathrm{Be}^{7 *}$ should also be about half of the total $\mathrm{Be}^{7}$ production; and because $\mathrm{Be}^{7}$ and $\mathrm{Li}^{7}$ are produced by mirror reactions their production cross sections should be about equal, even though no data for $\mathrm{Be}^{7}$ production in $\alpha \alpha$ reactions is available.

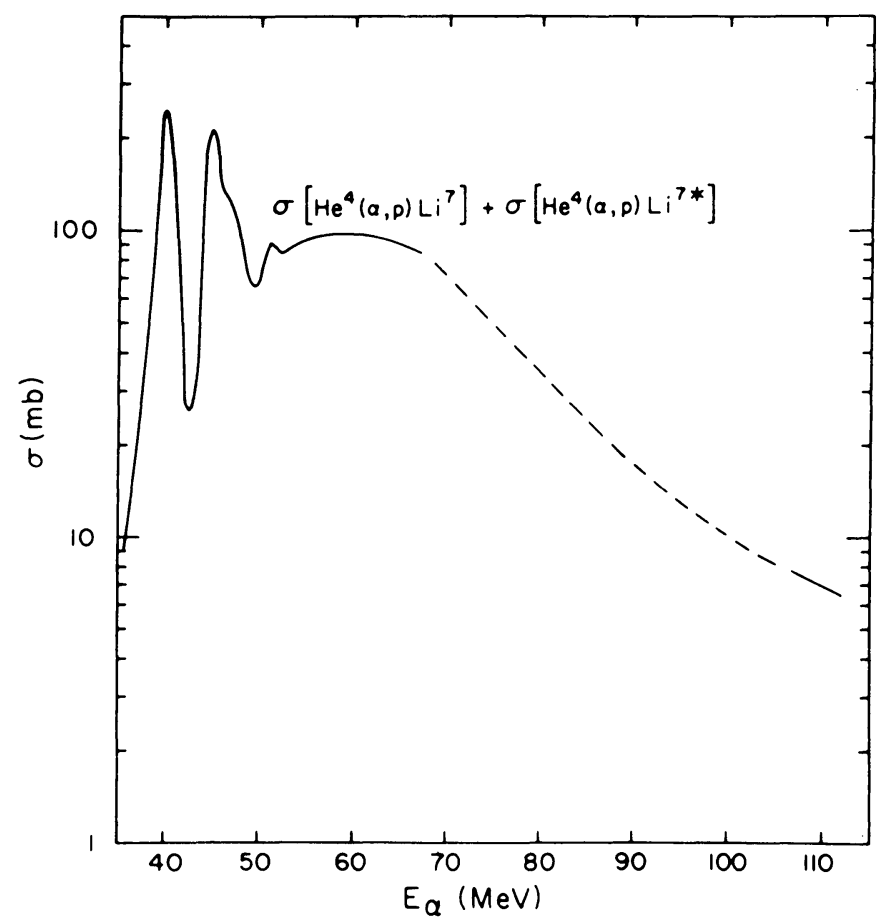

Fig. 9. $\mathrm{Li}^{7}$ production cross-section in $\alpha x$ reactions.

Using these cross-sections we find that the intensities of the lines at $478 \mathrm{keV}$ and $431 \mathrm{keV}$ are approximately the same as the intensity of the $4.43 \mathrm{MeV}$ line. Their Doppler width, however, are about $30 \mathrm{keV}$ (Kozlovsky and Ramaty, 1974a); therefore, they should not be observed individually but rather as a broad spectral feature.

The cross section for the other lines of Table II and their intensities were discussed by Ramaty and Lingenfelter (1973a). The intensities of these lines are significantly lower than the intensity of the $4.43 \mathrm{MeV}$ line for all interaction models and spectra.

\section{Accelerated Particles at the Sun and in the Interplanetary Medium}

The energy spectrum of the accelerated particles at the Sun can be deduced by comparing the calculated and observed ratios of the intensities of the strongest prompt line at $4.43 \mathrm{MeV}$ to that of the neutron capture line at $2.23 \mathrm{MeV}$. This ratio for both 
the thin- and thick-target models is shown in Figure 10 as a function of $s$, for powerlaw spectra, and $P_{0}$, for exponential spectra. These were obtained from the calculations of Sections III and IV using a photon yield, $f$, of 0.2 . This yield corresponds to a photospheric ratio $\mathrm{He}^{3} / \mathrm{H}=0$. For $\mathrm{He}^{3} / \mathrm{H}=5 \times 10^{-5}$ the curves in Figure 10 should be raised by about a factor of 2 . The $4.43 \mathrm{MeV}$ line is expected to be somewhat stronger than the $6.14 \mathrm{MeV}$ line. Since the same observational limits were reported for

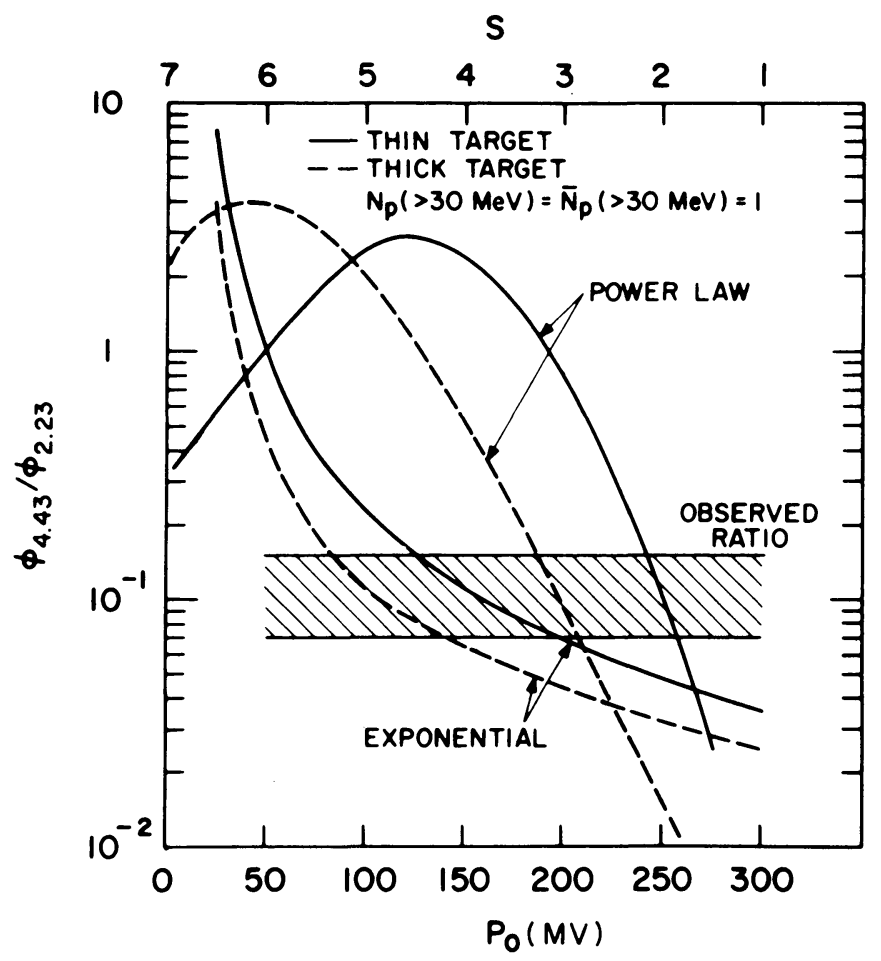

Fig. 10. Ratios of the $4.43 \mathrm{MeV}$ line intensity to the $2.23 \mathrm{MeV}$ line intensity for thin-and thick-target models, and power-law and exponential spectra.

these two lines, if we normalize our calculations to the $4.43 \mathrm{MeV}$ line, our results will also be consistent with the $6.14 \mathrm{MeV}$ line within the uncertainties of the measurements.

As can be seen from Figure 10 for exponential spectra in both the thin and thicktarget models $\phi_{4.43} / \phi_{2.23}$ decreases with increasing $P_{0}$. This results from the increase of the neutron production cross-section with increasing energy as opposed to the decrease of the excitation cross-sections (compare Figures 1 and 7). The same behavior can be seen for power-law spectra for $s$ smaller than about 4.5 in the thin-target model and $s$ less than about 6 in the thick-target model. For larger values of $s, \phi_{4.43} / \phi_{2.23}$ decreases with increasing $s$ because these neutrons are produced mainly in $\alpha \mathrm{CNO}$ and $\mathrm{CNO} \alpha$ reactions which have lower thresholds than those for prompt gamma-ray production. 
Let us compare now the calculations with the data. The observed (Chupp et al., 1975) $\phi_{4.43} / \phi_{2.23}$ ratio of $0.11 \pm 0.04$ for the 1972, August 4 flare is also shown in Figure 10. As can be seen from this figure, assuming power-law spectra for the particles in the flare region, their spectral index $s$ should lie between the values of $2 \pm 0.2$ deduced for the thin-target model and $3 \pm 0.3$ for the thick-target model if there is no $\mathrm{He}^{3}$ in the photosphere; or between $1.8 \pm 0.2$ and $2.7 \pm 0.2$ for these models if the photospheric $\mathrm{He}^{3} / \mathrm{H}$ ratio is $5 \times 10^{-5}$. Similarly, assuming exponential spectra, the implied $P_{0}$ 's should lie between $110 \pm 30 \mathrm{MV}$ for the thick-target model and $160 \pm 35 \mathrm{MV}$ for the thin target model if $\mathrm{He}^{3} / \mathrm{H}=0$; or between $180 \pm 50 \mathrm{MV}$ and $230 \pm 50 \mathrm{MV}$ for these models if $\mathrm{He}^{3} / \mathrm{H}=5 \times 10^{-5}$.

Comparison of these implied particle spectra in the solar flare region with the proton spectrum observed in the interplanetary medium from the 1972, August 4 flare is complicated by the possibility that the latter spectrum may have been significantly modified by acceleration in the interplanetary medium. Nonetheless, the proton spectrum obtained by Bertsch et al. (1974) from a rocket flight at 1916 UT 1972, August 4 had a spectral index $s \simeq 2$ in the 10 to $100 \mathrm{MeV}$ region, in good agreement with our conclusions.

Knowing the spectral index for the various models, we can now deduce the total number of protons at the Sun. In the thick-target model, the time integrated photon flux from the flare determines the total number of accelerated particles that interact and stop at the Sun. According to Chupp et al. (1975), the $2.2 \mathrm{MeV}$ intensity for the 1972, August 4 flare was about 0.3 photons $\mathrm{cm}^{-1} \mathrm{~s}^{-2}$. This flux is the average over the time interval of observation ( 0623 to $0633 \mathrm{UT}$ ) which gives a total flux of about 180 photons $\mathrm{cm}^{-2}$. Since the detector on OSO-7 was eclipsed by the Earth before the termination of the gamma-ray event, this is a lower limit to the total flux from the flare. If we assume a duration of $\sim 10^{3}$ seconds for the event, as indicated by the hard X-ray data (van Beek et al., 1973), then the total flux of $2.2 \mathrm{MeV}$ photons was $\sim 300 \mathrm{~cm}^{-2}$. From the neutron yield $Q$ shown in Figure 4 , we find that if $f=0.2$, $\bar{N}_{\mathrm{p}}(>30 \mathrm{MeV})=7 \times 10^{32}$ for $s=3$, and $\bar{N}_{\mathrm{p}}(>30 \mathrm{MeV})=1.0 \times 10^{33}$ for $P_{0}=110 \mathrm{MV}$. The corresponding values for $\mathrm{He}^{3} / \mathrm{H}=5 \times 10^{-5}$ are about the same, because the effect of a lower $f$ is approximately cancelled by a higher neutron yield. Therefore, the total number of protons above $30 \mathrm{MeV}$ released downward into the Sun in the thick-target model is about $10^{33}$ independent of the spectral form or the $\mathrm{He}^{3} / \mathrm{H}$ ratio.

For the thin-target model, the instantaneous gamma-ray observations determine the product of the ambient proton or hydrogen density, $n_{\mathrm{H}}$, and the instantaneous number of the accelerated particles in the interaction region. From the neutron yield $q$ shown in Figure 4, the flux given above implies that if $f=0.2, n_{\mathrm{H}} N_{\mathrm{p}}(>30 \mathrm{MeV}) \simeq 5 \times 10^{43}$ $\mathrm{cm}^{-3}$ for $s=2$, and $n_{\mathrm{H}} N_{\mathrm{p}}(>30 \mathrm{MeV}) \simeq 10^{44} \mathrm{~cm}^{-3}$ for $P_{0}=160 \mathrm{MV}$. As before, these values are not affected by the photospheric $\mathrm{He}^{3} / \mathrm{H}$ ratio.

To obtain an estimate of the number of protons released into the interplanetary medium, the information obtained from the gamma rays in the thin-target model can be combined with data on the path length traversed by the nuclei before their escape from the interaction region. Such information can be obtained from studies of deuter- 
ons and helium-3 nuclei from flares. The $\mathrm{H}^{2}$ and $\mathrm{He}^{3}$ observations from the 1972, August events (Webber et al., 1974), when compared with calculations of the production of these isotopes in nuclear reactions (Ramaty and Kozlovsky, 1974) imply that the amount of matter traversed by relativistic particles is about $1.5 \mathrm{~g} \mathrm{~cm}^{-2}$. This means that the product $n_{\mathrm{H}} t_{1}$ is about $3 \times 10^{13} \mathrm{~cm}^{-3} \mathrm{~s}$, where $t_{1}$ is the interaction time of the particles at the Sun. If $t_{1}$ is also interpreted as the escape time of the particles from the interaction region, then the protons were released into the interplanetary medium at an average rate $N_{\mathrm{p}}(>30 \mathrm{MeV}) / t_{1}$ varying from about 1.6 to $3 \times 10^{30}$ protons $^{-1}$. The total number of protons released is the product of this rate and the acceleration time $T$. As indicated by the X-ray data (van Beek et al., 1973), $T$ is about $10^{3} \mathrm{~s}$; therefore the total number of protons released is between about 1.6 to $3 \times 10^{33}$ protons. These numbers are larger by only about a factor of 2 to 3 than the number of protons released downward into the Sun in the thick-target model.

The number of protons released from the flare should be compared with estimates of the total number of protons in the interplanetary medium as obtained from charged particle observations. Ramaty and Lingenfelter (1973a) have estimated this quantity by taking the observed density of protons near Earth and by multiplying it with a storage volume in the interplanetary medium which they took to be $\sim 10^{39} \mathrm{~cm}^{3}$. By using this volume and the peak proton flux greater than $30 \mathrm{MeV}$ measured by Kohl et al. (1973), we get about $10^{34}$ protons. This number is larger by a factor of 3 to 6 than our estimate for the number of protons released in the thin-target model. But since the measured peak proton flux could consist to a large degree of particles accelerated by shocks in the interplanetary medium, it appears that there is no real discrepancy between the number of protons released from the Sun as deduced from the gamma rays and the number observed in interplanetary space.

\section{The Nature of the Positron Annihilation Radiation}

Positrons can be produced in solar flares from the decay of $\pi^{+}$mesons and radioactive nuclei which result from nuclear reactions of accelerated protons and nuclei with the ambient medium. The possibility that a significant fraction of solar positrons can also be produced by accelerated electrons in $\mathrm{e}^{+}-\mathrm{e}^{-}$pairs is being considered by Bai and Ramaty (1975). Annihilation of positrons can produce a gamma-ray line at $0.51 \mathrm{MeV}$. This line was observed by Chupp et al. (1973) for both the 1972, August 4 and August 7 flares.

The cross sections for the production of $\pi^{+}$mesons and $\beta^{+}$-emitting radioactive nuclei were given by Lingenfelter and Ramaty (1967). Kozlovsky (1974, private communication) has recently updated the cross sections of the $\beta^{+}$-emitters. Using these cross-sections we calculate from equation (3) the instantaneous production rates of positron-emitters in the thin-target model for power-law spectra. The results are shown in Figure 11. As can be seen, for flatter spectra the principal positron source is $\pi^{+}$mesons, but for steeper spectra, the pion contribution is negligible. This effect can also be seen in Figure 12, where the ratio of the pion yield to the total positron-emitter 


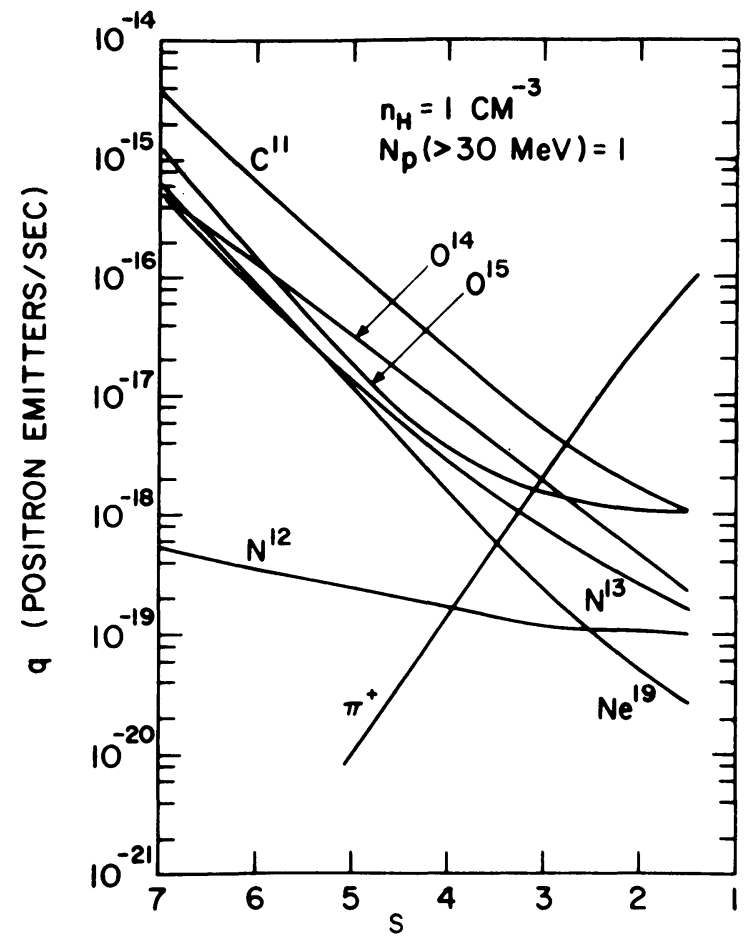

Fig. 11. Partial positron production modes in the thin-target model with power-law spectra.

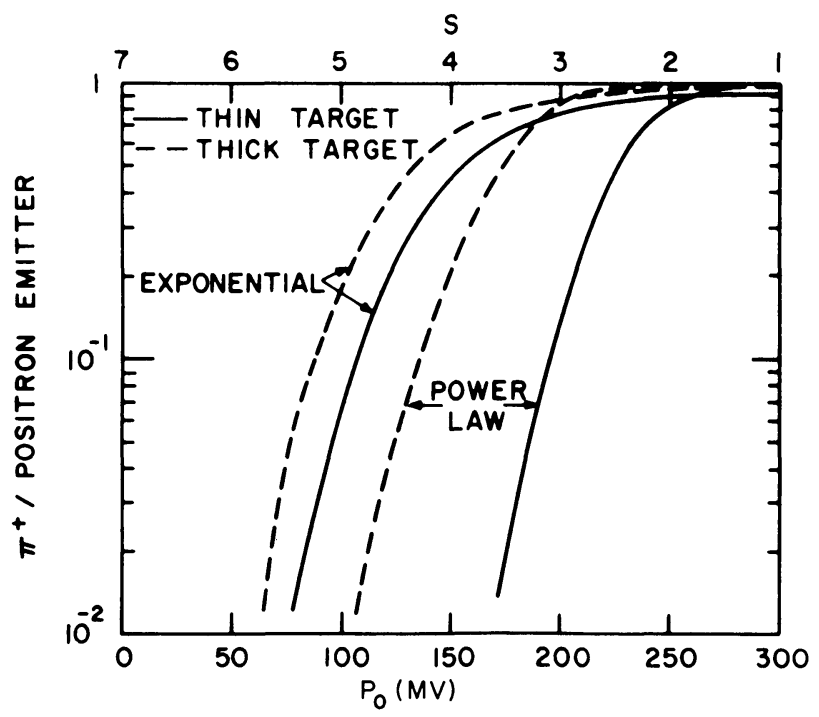

Fig. 12. Ratios of the $\pi$-meson yields to the total positron yields for the thin-and thick-target models, and power-law and exponential spectra. 
yield (including both pions and radioactive nuclei) is plotted as a function of $s$ or $P_{0}$ for both interaction models. We see that, as for neutron production, the efficiency of $\pi^{+}$-production is larger in the thick-target model than in the thin-target model. The ratios of the total yield of positron-emitters to the neutron yield are shown in Figure 13 for the various interaction models.

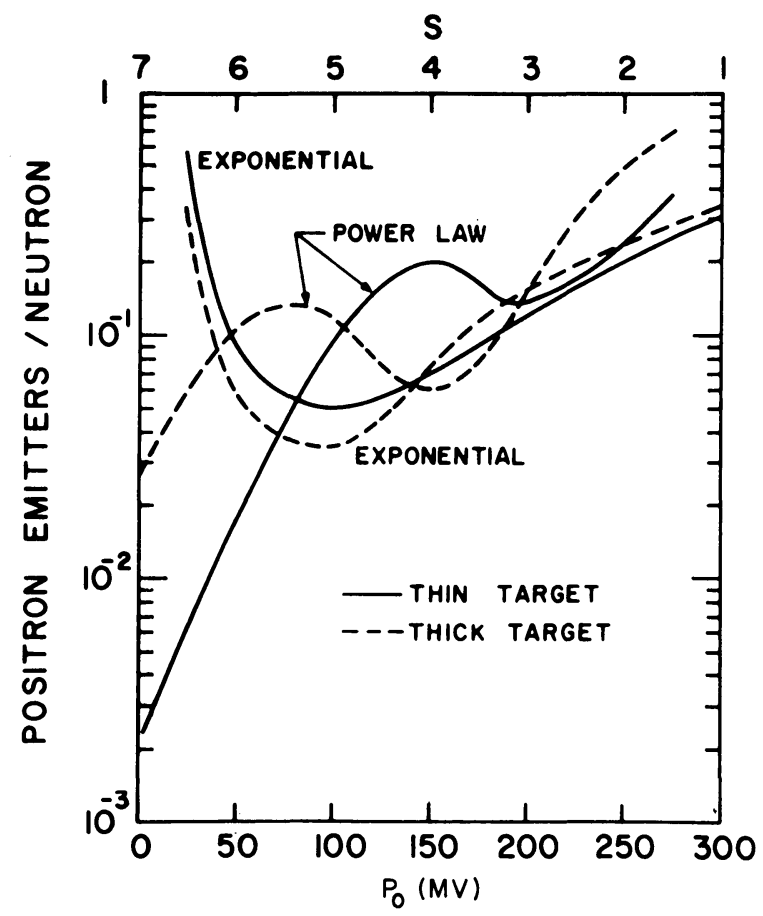

Fig. 13. Ratios of the total positron yield to the total neutron yield for the thin- and thick-target models, and power-law and exponential spectra.

TABLE III

\begin{tabular}{ll} 
& $\begin{array}{l}\text { Positron emitter } \\
\text { mean lives (s) }\end{array}$ \\
\hline$\pi^{+}$ & $2.6 \times 10^{-8}$ \\
$\mathrm{~N}^{12}$ & 0.016 \\
$\mathrm{C}^{11}$ & 1766 \\
$\mathrm{~N}^{13}$ & 863 \\
$\mathrm{O}^{14}$ & 102 \\
$\mathrm{O}^{15}$ & 176 \\
\hline
\end{tabular}

The intensity of the $0.51 \mathrm{MeV}$ line, however, depends not only on the number of positron emitters produced, but also on the decay rate of the positron emitters and on the annihilation rate of the positrons. The mean lives against radioactive decay of the various positron emitters are shown in Table III. 
The annihilation rate of the positrons depends on the density, temperature and state of ionization of the ambient medium. As discussed previously (Stecker, 1969; Ramaty and Lingenfelter, 1973b) in a low density and neutral medium, positrons nearly always annihilate via positronium formation; only if the ambient density exceeds about $10^{15} \mathrm{~cm}^{-3}$ do collisions destroy the ${ }^{3} \mathrm{~S}$ state of positronium at such a rate that free annihilation becomes more important than positronium annihilation (Leventhal, 1973). Positron annihilation from a bound state of positronium results in an asymmetric $0.51 \mathrm{MeV}$ line, since $75 \%$ of the time positronium annihilated from the ${ }^{3} \mathrm{~S}$ state into 3 photons of energies less than $0.51 \mathrm{MeV}$, instead of 2 photons at precise-

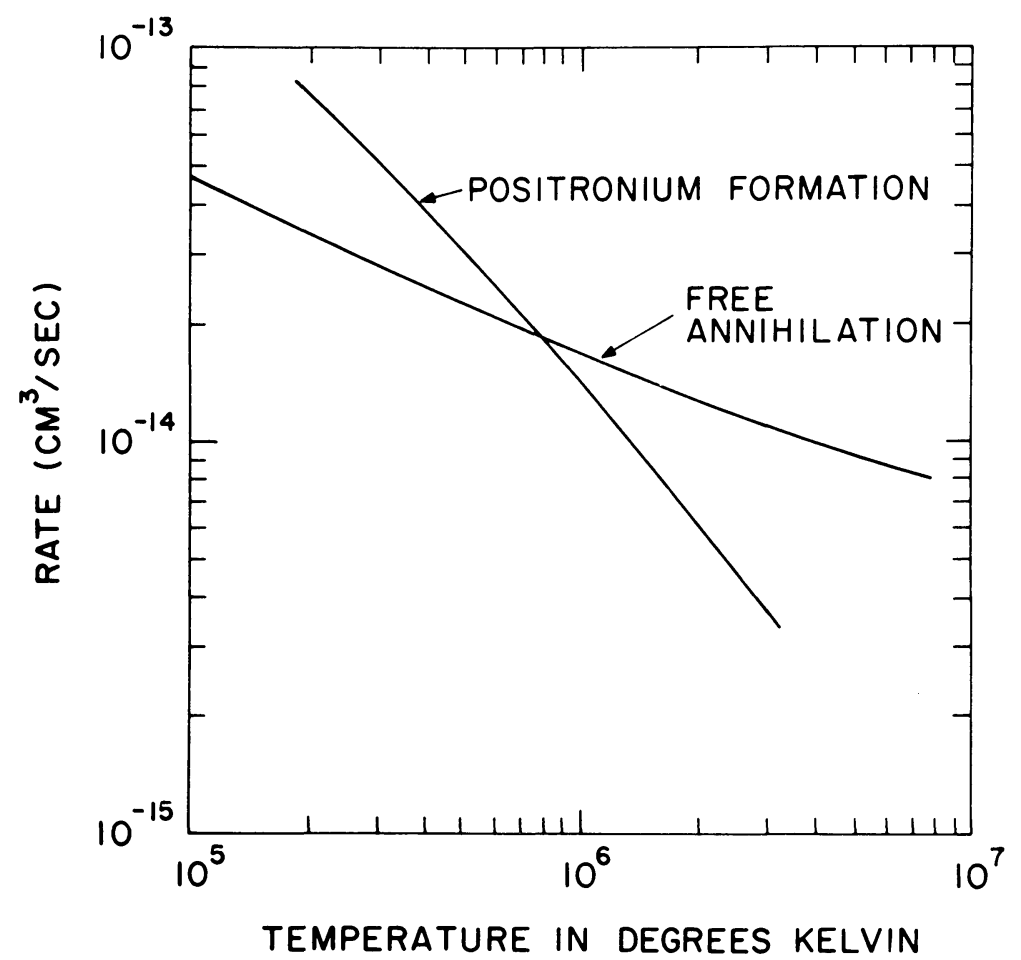

Fig. 14. Positron free-annihilation rate and positronium formation rate in a hydrogen plasma.

ly this energy. The $0.51 \mathrm{MeV}$ line from the Sun, however, appears to be symmetric, and the observational upper limit on the fraction of three-photon annihilations is about $20 \%$ of the total (D. Forrest, 1973, private communication).

As we noted, three-photon annihilation could be reduced by collisions, but the required density of $\gtrsim 10^{15} \mathrm{~cm}^{-3}$ is quite large. However, if the ambient medium is ionized, the rate of positronium formation is greatly reduced. In Figure 14 we show the rate of positronium formation and free annihilation per positron in a hydrogen plasma of unit density as a function of its temperature (C. Werntz and C. Crannell, 1973, private communication). 
As can be seen, if the temperature is greater than about $10^{6} \mathrm{~K}$, most of the positrons annihilate without forming positronium. This is not an unreasonable temperature for the annihilation region; however, from the observed upper limit on the width of the $0.51 \mathrm{MeV}$ line, the temperature in the annihilation region should be less than $\sim 10^{7} \mathrm{~K}$ (Chupp et al., 1975).

According to the observations of the 1972, August 4 flare, the rise time of the 0.51 $\mathrm{MeV}$ line was within instrumental errors, similar to or perhaps even shorter than the rise time of the $2.23 \mathrm{MeV}$ line (Chupp et al., 1975). The latter was about $100 \mathrm{~s}$, consistent with the expected lag between the production of the neutrons and the formation of the $2.2 \mathrm{MeV}$ line (see Table I). Assuming that the time dependence of the production rate of the positron emitters is the same as that of the neutrons, the lag between the production of the positron emitters and the formation of the $0.51 \mathrm{MeV}$ line should not be longer than about $100 \mathrm{~s}$. From Figure 14, this result implies that the density of the ambient medium in the annihilation region is at least $10^{12} \mathrm{~cm}^{-3}$. Furthermore if the positrons are of nuclear origin, then from Table III it follows that they should mainly result from $\pi^{+}$-mesons and short lived radioactive nuclei $\left(\mathrm{O}^{15}, \mathrm{O}^{14}\right.$ and $\mathrm{N}^{12}$ ).

The observed average flux in the $0.51 \mathrm{MeV}$ line for the flare of 1972, August 4 was about 0.06 photons $\mathrm{cm}^{-2} \mathrm{~s}^{-1}$ (Chupp, 1975). Hence the observed $\phi_{0.51} / \phi_{2.23}$ ratio was about 0.2 .

Let $f^{\prime}$ be the $0.51 \mathrm{MeV}$ photon yield per positron defined in the same way as the $2.23 \mathrm{MeV}$ photon yield per neutron, $f$, in Equation (9). As discussed in Section III, $f$ ranges from about 0.1 to 0.2 . The maximum value of $f^{\prime}$ is 2 . But because part of the positrons can escape from the Sun before they annihilate, and, furthermore, a fraction of the positrons can be trapped at the Sun in low density regions where the annihilation time is long, $f^{\prime}$ can be considerably less than 2 .

According to our discussion in Section V, for power-law spectra $s \simeq 2$ in the thintarget model, and $s \simeq 3$ in the thick-target model. For these spectral parameters, from Figure 12 we see that the bulk of the positron emitters are $\pi^{+}$-mesons, and from Figure 13 we get that the positron emitter-to-neutron ratio is about 0.2 . The observed ratio, $\phi_{0.51} / \phi_{2.23} \simeq 0.2$ then implies that $f^{\prime} \simeq f$, i.e. the $0.51 \mathrm{MeV}$ yield per positron is about the same as the $2.23 \mathrm{MeV}$ yield per neutron. In view of the uncertainties involved in the deductions of $f^{\prime}$, this is not an unreasonable result. We can also deduce $f^{\prime}$ for the various values of $P_{0}$ obtained in Section $\mathrm{V}$, and in all cases we find acceptable values $\left(0.1<f^{\prime}<2\right)$. For these exponential spectra the contribution of $\pi^{+}$-mesons to the total positron production is greater than about $50 \%$ in all cases, except in the thicktarget model with no $\mathrm{He}^{3}$ in the photosphere where they contribute only about $25 \%$ of the positrons. A definite test for the possibility that the bulk of the positrons are due to $\pi^{+}$-mesons would be the observation of gamma rays from $\pi^{0}$ decay. As there is no published data on high energy solar gamma rays, we shall not discuss this possibility in the present paper.

As mentioned above, prompt $0.51 \mathrm{MeV}$ photons could also result from positrons produced in $\mathrm{e}^{+}-\mathrm{e}^{-}$pairs. Such pairs would be produced mainly by $\geq 1 \mathrm{MeV}$ elec- 
trons. Because these electrons also produce continuum emission by bremsstrahlung, it is possible to calculate the pair-to-bremsstrahlung yield (Bai and Ramaty, 1975). By comparing these calculations with the observed continuum emission from the 1972, August 4 flare (Chupp et al., 1975), we find that if the bremsstrahlung is produced by an isotropic distribution of electrons at the Sun, pair production could account for less than $1 \%$ of the positrons required to produce the $0.51 \mathrm{MeV}$ line. However, if the electron beam is directed downward into the Sun, the bremsstrahlung efficiency in the backward direction is greatly reduced for relativistic particles (Petrosian, 1973). In this case pair production could account for essentially all the prompt $0.51 \mathrm{MeV}$ photons of the 1972, August 4 flare.

Finally, we wish to mention that delayed photons (by about $15 \mathrm{~min}$ ) were observed for the 1972, August 7 flare (Chupp et al., 1973). These photons are most likely due to the long lived radioactive nuclei such as $\mathrm{C}^{11}$ and $\mathrm{N}^{13}$.

\section{Summary}

The observed gamma-ray lines from the 1972, August 4 flare, at 0.5, 2.2, 4.4 and 6.1 MeV are due to positron annihilation, neutron capture on hydrogen, and deexcitation of excited states in $\mathrm{C}^{12}$ and $\mathrm{O}^{16}$, respectively. The strongest line is at $2.23 \mathrm{MeV}$. It is due to fast neutrons produced by nuclear reactions of flare accelerated particles with the ambient solar atmosphere. These neutrons are thermalized and captured by ambient protons in the photosphere to produce deuterons and $2.23 \mathrm{MeV}$ gamma rays. Photospheric $\mathrm{He}^{3}$ competes with the protons in capturing neutrons. Because captures on $\mathrm{He}^{3}$ do not lead to photon emission, the observation of $2.23 \mathrm{MeV}$ line emission from the Sun implies that the $\mathrm{He}^{3}$ abundance in the photosphere cannot be much larger than that observed in the solar wind $\left(\mathrm{He}^{3} / \mathrm{H} \sim 5 \times 10^{-5}\right)$.

We have evaluated in detail the yield of neutrons and excited $\mathrm{C}^{12}$ and $\mathrm{O}^{16}$ nuclei from nuclear reactions of accelerated particles with the ambient solar atmosphere. For the 1972, August 4 flare the neutrons are produced mainly in $p \alpha$ and $\alpha p$ reactions by primary particles with energies greater than about $30 \mathrm{MeV}$ nucleon $^{-1}$. The observed gamma rays at $4.43 \mathrm{MeV}$ and $6.14 \mathrm{MeV}$ are principally due to proton induced interactions. Reactions induced by fast carbon and oxygen nuclei lead to Dopplerbroadened lines which cannot be distinguished from the continuum.

From the comparison of the calculated and observed ratios of the lines at $4.43 \mathrm{MeV}$ or $6.14 \mathrm{MeV}$ to the line at $2.23 \mathrm{MeV}$ it is possible to deduce the spectrum of the accelerated particles in the flare region. The spectral index $s$ defined in Equation (1) is $2 \pm 0.2$ for the thin-target model and $3 \pm 0.3$ for thick-target model if there is no $\mathrm{He}^{3}$ in the photosphere; or $1.8 \pm 0.2$ and $2.7 \pm 0.2$ for these models if the photospheric $\mathrm{He}^{3} / \mathrm{H}$ ratio is $5 \times 10^{-5}$. The characteristic rigidity $P_{0}$ defined in Equation (2) is $160 \pm 35 \mathrm{MV}$ for the thin-target model and $110 \pm 30 \mathrm{MV}$ for the thick-target model if $\mathrm{He}^{3} / \mathrm{H}=0$; or $230 \pm 50 \mathrm{MV}$ and $180 \pm 50 \mathrm{MV}$ for these models if $\mathrm{He}^{3} / \mathrm{H}=5 \times 10^{-5}$. The total number of protons above $30 \mathrm{MeV}$ released downward into the Sun in the thick-target model is about $10^{33}$. For the thin-target model about $2 \times 10^{33}$ protons escape from the flare 
region if the thickness of the target for relativistic particles is $1.5 \mathrm{~g} \mathrm{~cm}^{-2}$ as deduced from deuteron and helium-3 observations.

The positrons which produce the $0.51 \mathrm{MeV}$ line could be due to $\pi^{+}$-mesons, radioactive nuclei, and pair production. We have evaluated in detail the production of mesons and radioactive nuclei in nuclear reactions of accelerated charged particles with ambient nuclei. The relatively prompt nature of the $0.51 \mathrm{MeV}$ line seems to favor positron production from $\pi^{+}$-decay or $\mathrm{e}^{+}-\mathrm{e}^{-}$pairs in the initial phase of the 1972 , August 4 event. The delayed $0.51 \mathrm{MeV}$ line emission observed for the 1972, August 7 event is very likely due to positrons from radioactive nuclei.

\section{Acknowledgements}

The research of REL was supported by the National Science Foundation under Grant GP 31620.

\section{References}

Bai, T. and Ramaty, R.: 1975 (to be published).

Bertsch, D. L., Biswas, S., and Reames, D. V.: 1974, Solar Phys. 39, 479.

Cameron, A. G. W.: 1973, Space Sci. Rev. 15, 121.

Chupp, E. L., Forrest, D. J., and Suri, A. N.: 1975, This volume, p. 341.

Chupp, E. L., Forrest, D. J., Higbie, P. R., Suri, A. N., Tsai, C., and Dunphy, P. P.: 1973, Nature 241, 333.

Forrest, D.: 1973, Private communication.

Kohl, J. W., Bostrom, C. O., and Williams, D. J.: 1973, in H. E. Coffey (ed.), World Data Center Rept. UAG 28, Part II, Collected Data Reports on August 1972 Solar Terrestrial Events, p. 330. Kożlovsky, B.: 1974, Private communication.

Kozlovsky, B. and Ramaty, R.: 1974a, Astrophys. J. Letters 191, L43.

Kozlovsky, B. and Ramaty, R.: 1974b, Astron. Astrophys. 34, 477.

Leventhal, M.: 1973, Astrophys. J. Letters 183, L147.

Lingenfelter, R. E. and Ramaty, R.: 1967, in B. S. P. Shen (ed.), High Energy Nuclear Reactions in Astrophysics, W. A. Benjamin, New York, p. 99.

Lingenfelter, R. E., Flamm, E. J., Canfield, E. H., and Kellman, S. : 1965, J. Geophys. Res. $70,4077$. Petrosian, V.: 1973, Astrophys. J. 186, 291.

Ramaty, R. and Kozlovsky, B.: 1974, Astrophys. J. (in press).

Ramaty, R. and Lingenfelter, R. E.: 1973a, in R. Ramaty and R. G. Stone (eds.), High Energy Phenomena on the Sun, NASA SP-342, p. 301.

Ramaty, R. and Lingenfelter, R. E.: 1973b, Conference Papers, 13th International Cosmic Ray Conference, University of Denver, Colorado, p. 1590.

Reppin, C., Chupp, E. L., Forrest, D. J., and Suri, A. N. : 1973, Conference Papers, 13th International Cosmic Ray Conference, University of Denver, Denver, Colorado, p. 1577.

Stecker, F. W.: 1969, Astrophys. Space Sci. 3, 479.

Van Beek, H. F., Hoyng, P. and Stevens, G. A.: 1973, in H. E. Coffey (ed.), World Data Center, Rept. UAG 28, Part II, Collected Data Reports on August 1972 Solar Terrestrial Events, p. 319. Wang, H. T. and Ramaty, R.: 1974, Solar Phys. 36, 129.

Webber, W. R., Roelof, E. C., McDonald, F. B., Teegarden, B. J., and Trainor, J.: 1974 (preprint). Werntz, C. and Crannell, C.: 1973, Private communication. 